\title{
Lincosamide (Clindamycin)
}

\author{
M. Fille, S. Ziesing \\ S. Suerbaum et al. (Hrsg.), Medizinische Mikrobiologie und Infektiologie, \\ DOI 10.1007/978-3-662-48678-8_101, @ Springer-Verlag Berlin Heidelberg 2016
}

Clindamycin ist ein Lincosamidantibiotikum. Es wirkt bakteriostatisch auf grampositive aerobe und obligat anaerob wachsende Bakterien.

\section{Steckbrief}

Clindamycin ist ein Lincosamidantibiotikum. Es wirkt bakteriostatisch auf grampositive aerobe und obligat anaerob wachsende Bakterien.

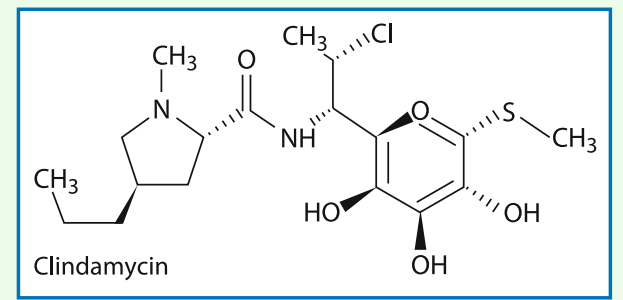

\subsection{Beschreibung}

\section{- Wirkungsmechanismus und Wirktyp}

Clindamycin hemmt die Proteinbiosynthese in der Bakterienzelle.

\section{- Wirkungsspektrum}

Clindamycin wirkt gut gegen Staphylokokken und Anaerobier, auch gegen Pneumokokken, Streptokokken und Diphtheriebakterien. Anaerobe Bakterien wie Bacteroides-, Fusobacterium- und Actinomyces-Arten, Peptostrepto- und Peptokokken, außerdem Propionibakterien und die meisten C. perfringens-Stämme werden erfasst. Es wirkt auch gegen Pneumocystis, Plasmodien und Toxoplasmen.

Resistent sind C. difficile, ebenso Enterokokken, Mykoplasmen und sämtliche aeroben gramnegativen Stäbchenbakterien.

\section{- Pharmakokinetik}

Clindamycin wird nach oraler Gabe gut resorbiert und kann auch i. v. appliziert werden. Die Halbwertszeit beträgt ca. 3 h. Es hat eine gute Gewebegängigkeit und penetriert das Knochengewebe. Clindamycin wird in der Leber metabolisiert und nur zu $30 \%$ in aktiver Form mit dem Harn ausgeschieden.

\section{- Resistenz}

Die Resistenz entsteht durch Veränderungen der Bindungsstellen am Ribosom.

\subsection{Rolle als Therapeutikum}

\section{- Indikationen}

Anaerobierinfektionen und schwere Staphylokokkeninfektionen bei Patienten mit Penicillinallergie stellen die häufigsten Indikationen dar. Kombinationen mit $\beta$-Laktam-Antibiotika erweitern das Spektrum bei komplizierten, polymikrobiellen Infektionen.

Eine weitere Indikation stellt die Verwendung bei lebensbedrohlichen Infektionen mit grampositiven, toxin- oder superantigenproduzierenden Erregern dar, bei denen der Wirkungsmechanismus als Proteinbiosynthese-Inhibitor zur Suppression der Toxinproduktion genutzt wird.

Infektionen mit ambulant erworbenen (»community-acquired «) MRSA-Stämmen können meist mit Clindamycin therapiert werden.

\section{- Kontraindikationen}

In Schwangerschaft und Stillperiode soll Clindamycin nicht gegeben werden; da die i. v. Präparation verhältnismäßig viel Benzylalkohol enthält, verbietet sich auch der Gebrauch im 1. Lebensmonat wegen möglicher schwerer Atemstörungen und Angioödemen.

\section{- Anwendungen}

Clindamycin kann sowohl oral als auch parenteral verabreicht werden.

\section{- Nebenwirkungen}

Eine gefährliche Nebenwirkung ist die bei Erwachsenen häufiger als bei Kindern auftretende antibiotikaassoziierte Kolitis, in ihrer schwersten Form die pseudomembranöse Enterokolitis, die durch Überwuchern clindamycinresistenter; toxinbildender C. difficileStämme ausgelöst werden kann. Allergische Reaktionen sind selten. Nach i. v. Gabe können Ikterus oder Leberfunktionsstörung auftreten.

\section{In Kürze}

Lincosamide (Clindamycin)

Resistenz Veränderte Bindungsstellen am Ribosom.

Indikationen Anaerobierinfektionen, schwere Staphylokokkeninfektionen bei Penicillinallergie, Toxoplasmose, PneumocystisPneumonie Kontraindikationen Schwangerschaft und Stillperiode, Neugeborene. 